\title{
An Alternative Bandwidth Selection Method for Estimating Functional Coefficient Models*
}

\author{
Xirong Chen ${ }^{\mathrm{a}, \mathrm{d}}$, Ta-Cheng Huang ${ }^{\mathrm{b}, \mathrm{d}}$, and Qi Li ${ }^{\dagger c, d}$ \\ ${ }^{a}$ School of Economics \& Management, Beihang University, China \\ ${ }^{\mathrm{b}}$ Department of Finance and Economics, Texas State University \\ ' International School of Economics \& Management, Capital University Economics \& \\ Business \\ ${ }^{\mathrm{d}}$ Department of Economics, Texas A\&M University
}

\begin{abstract}
Functional coefficient regression models are very useful for many statistics and economics applications and there exists a large body of literature on kernel estimation of the coefficient functions. Fan and Zhang (1999) point out that the traditional estimation method is not optimal if the coefficient functions possess different degrees of smoothness. They propose a two-step method to attenuate the drawback. To apply the two-step method, one needs to identify which coefficient function is the smoothest one and undersmooth all other functions in the first stage. In this paper we propose an alternative approach which assigns each smooth function a different bandwidth and we choose the bandwidths simultaneously. Simulation results show that our proposed method compare well with existing methods.

JEL classification: $C 13 ; C 14$
\end{abstract}

Keywords: Functional coefficient models, local polynomial estimation, bandwidth selection, mean squared errors

${ }^{*}$ We would like to thank a referee, a co-editor and Yiguo Sun for their helpful comments that greatly improve the paper. Li's research is partially supported by China National Science Foundation, projects' \# 71133001 and \# 71601130.

†The corresponding author: Qi Li. Email: qi-li@tamu.edu

(C) 2017. This manuscript version is made available under the Elsevier user license http://www.elsevier.com/open-access/userlicense/1.0/ 


\section{Introduction}

Functional-coefficient, also known as varying-coefficient, regression models are very useful for many statistics and economics applications, and in particular when additive separability of covariates is unsuitable for the problem at hand (Cai et al., 2006). For example, Card (2001) points out that the marginal returns to education are also plausibly increasing in work experience and, therefore, it is natural to model the marginal returns to education as a functional coefficient model. Other examples include Cai (2007) who estimate a timevarying $\beta$-coefficient function in a CAPM, and Li et al. (2002) who consider the estimation of a production function with marginal productivity depending firm's R\&D level. For theories and applications of varying coefficient models to non-stationary data, see Juhl (2005), Sun and Li (2011) and Xiao (2009).

In this paper, we consider the following functional-coefficient model

$$
Y=\sum_{\ell=1}^{L} g_{\ell}(Z) X_{\ell}+U
$$

for given covariates $\left(Z, X_{1}, \ldots, X_{L}\right)$ and the error term $U$ satisfies $\mathbb{E}\left(U \mid Z, X_{1}, \ldots, X_{L}\right)=0$. We only consider the scalar $Z$ case for notational simplicity. Note that model (1) is a special case of the functional-coefficient models, proposed by Hastie and Tibshirani (1993), which has the form

$$
\mathcal{L}(\mathbb{E}(Y \mid x, z))=\sum_{\ell=1}^{L} g_{\ell}(z) x_{\ell}
$$

where $\mathcal{L}(\cdot)$ is a known link function. There exists a large body of literature on estimating model (1) by kernel method, see Cai et al. (2000), Cai (2007) and the references therein.

As pointed out by Fan and Zhang (1999), when the coefficient functions have different degrees of smoothness, the conventional estimation method, which chooses one bandwidth for all coefficient functions, proposed by Cleveland et al. (1992), is suboptimal for the smoothest coefficient function. Fan and Zhang (1999) propose a two-step estimation procedure which estimates the smoothest function by local cubic estimator in the second step while all other coefficient functions are undersmoothed by using a bandwidth which has a smaller magnitude than the one chosen for the one-step estimation. They show that the local cubic estimator for the smoothest function can achieve the optimal rate. Cai (2002) extends the two-step estimation method to model (2) with a known link function. Cai et al. (2006) apply the two-step estimation procedure to the functional coefficient instrumental variables models. It is worth to note that in order to apply the two-step procedure to estimate the coefficient functions, one needs to know which coefficient function is the smoothest one among all functions. 
In this paper, we propose a new estimation method. Unlike the conventional approach that uses a common smoothing parameter to all coefficient functions, we assign a different bandwidth to each coefficient function and we choose these bandwidths simultaneously by minimizing a cross validated criterion function. Therefore, our method allows for different amount of smoothing to different coefficient functions. Moreover, compared to the two-step procedure, our procedure does not need to identify which function is the smoothest among all the coefficient functions.

The paper is organized as follows. In section 2 we discuss existing estimation procedures and introduce our proposed method. In section 3 we use simulations to examine the finite sample performance of our proposed method. Section 5 concludes.

\section{Bandwidth Selection Procedures}

The local $p$ th order polynomial kernel estimators for the coefficient functions in model (1), for given any point $z$, is based on minimizing the following objective function with respect to parameters $a_{j, \ell}$ for $j=0,1, \ldots, p$ and $\ell=1, \ldots, L$.

$$
\min _{\left\{a_{0, \ell}, a_{1, \ell}, \ldots, a_{p, \ell}\right\}_{\ell=1}^{L}} \sum_{i=1}^{n}\left\{Y_{i}-\sum_{\ell=1}^{L}\left[a_{0, \ell}-a_{1, \ell}\left(Z_{i}-z\right)-\cdots a_{p, \ell}\left(Z_{i}-z\right)^{p}\right] X_{i \ell}\right\}^{2} K_{h}\left(Z_{i}-z\right)
$$

where $K_{h}(\cdot)=K(\cdot / h) / h, K(\cdot)$ is a kernel function, and $h=h_{n}$ is a bandwidth. Note that $a_{0, \ell}$ estimates $g_{\ell}(z)$ and $a_{j, \ell}$, for $j \geq 1$, estimates $(j !) g_{\ell}^{(j)}(z)$ for $j=1, \ldots, p$, where $g_{\ell}^{(j)}(z)$ is the $j^{\text {th }}$ derivative of $g_{\ell}(z)$. Let $\widehat{g}_{\ell, h}(z ; p)=\widehat{a}_{0, \ell, h}(z ; p)$ denote the values of $a_{0, \ell}$, for $\ell=1,2, \ldots, L$, that minimize the above objective function. Then $\widehat{g}_{\ell, h}(\cdot ; p)$ estimates $g_{\ell}(\cdot)$. The solution to the previous problem can be written as a weighted least squares regression problem. Let $W_{i}(z ; p)=\left(X_{i 1}, X_{i 1}\left(Z_{i}-z\right), \ldots, X_{i 1}\left(Z_{i}-z\right)^{p}, \ldots, X_{i L}, X_{i L}\left(Z_{i}-z\right), \ldots, X_{i L}\left(Z_{i}-z\right)^{p}\right)$. Then, the local $p$ th order polynomial kernel estimator of $g_{\ell}(z), \ell=1, \ldots, L$, can be expressed as

$$
\begin{aligned}
\widehat{g}_{\ell, h}(z ; p)= & e_{(\ell-1)(1+p)+1, L \times(p+1)}\left[\sum_{i=1}^{n} K_{h}\left(Z_{i}-z\right) W_{i}^{\top}(z ; p) W_{i}(z ; p)\right]^{-1} \\
& \times\left[\sum_{i=1}^{n} K_{h}\left(Z_{i}-z\right) W_{i}^{\top}(z ; p) Y_{i}\right]
\end{aligned}
$$

where $e_{k, m}$ is the unit vector of length $m$ with 1 at the $k^{\text {th }}$ component and zeros for all other components, and the superscript $T$ denotes the transpose of a matrix.

Cleveland et al. (1992) propose using local linear estimator of $g_{\ell}(\cdot), \ell=1, \ldots, L$, with a bandwidth, $h_{1}$, which minimizes the following cross-validation (CV) function

$$
h_{1}=\operatorname{argmin}_{h} C V_{1}(h)=\operatorname{argmin}_{h} \frac{1}{n} \sum_{i=1}^{n}\left[Y_{i}-\sum_{\ell=1}^{L} \widehat{g}_{\ell, h,-i}\left(Z_{i} ; 1\right) X_{i \ell}\right]^{2},
$$


where

$$
\begin{aligned}
\widehat{g}_{\ell, h}(z ; p)= & e_{(\ell-1)(1+p)+1, L \times(p+1)}\left[\sum_{j \neq i}^{n} K_{h}\left(Z_{j}-Z_{i}\right) W_{j}^{\top}\left(Z_{i} ; p\right) W_{j}\left(Z_{i} ; p\right)\right]^{-1} \\
& \times\left[\sum_{j \neq i}^{n} K_{h}\left(Z_{j}-Z_{i}\right) W_{j}^{\top}\left(Z_{i} ; p\right) Y_{i}\right]
\end{aligned}
$$

Fan and Zhang (1999) point out that this estimation method implicitly assume that the coefficient functions posses about the same degrees of smoothness. They show that the estimator for the smoothest coefficient function with the bandwidth $h_{1}$ is not optimal. To attenuate the this drawback, Fan and Zhang (1999) propose a two-step procedure to estimate the functional coefficients which process different degrees of smoothness.

Without loss of generality, we assume that $g_{L}(\cdot)$ is smoother than $g_{\ell}(\cdot), \ell=1, \ldots, L-1$. The two-step procedure first local linearly estimates $g_{\ell}(\cdot), \ell=1, \ldots, L-1$ with a bandwidth $h_{0}=\alpha h_{1}, \alpha \in(0,1)$. It is worth to note that such a small enough bandwidth is needed for the bias of the estimators $\widehat{g}_{\ell}(\cdot), \ell=1, \ldots, L-1$, being negligible. Then, in the second step, $g_{L}(\cdot)$ is estimated by using a local cubic fit with the bandwidth $h_{2}$ which minimizes the following $\mathrm{CV}$ function

$$
h_{2}=\operatorname{argmin}_{h} C V_{2}(h)=\operatorname{argmin}_{h} \frac{1}{n} \sum_{i=1}^{n}\left[Y_{i}-\sum_{\ell=1}^{L-1} \widehat{g}_{\ell, h_{0}}\left(Z_{i} ; 1\right) X_{i \ell}-\widehat{g}_{L, h,-i}\left(Z_{i} ; 3\right) X_{i L}\right]^{2},
$$

where $\widehat{g}_{\ell, h_{\ell},-i}\left(Z_{i} ; 1\right)$ and $\widehat{g}_{\ell, h_{\ell},-i}\left(Z_{i} ; 3\right)$ are defined in $(4)$ with $p=1$ and 3 , respectively.

Fan and Zhang (1999) shows that the two-step estimator can achieve the optimal convergent rate for estimating the smoothest coefficient function. They also point out that if $g_{L}(\cdot)$ is only as smooth as the other functions, the estimators of the two-step procedure will have the same asymptotic bias and variance as of the one-step method provided the first stage bandwidth $h_{0}$ is small enough; that is, the two-step procedure achieve the same convergence rate as the one-step method.

The two-step procedure requires that the smoothest coefficient function is fitted again at the second stage by replacing other less smooth functions with their undersmoothed estimates. In practice, to apply the two-step procedure, one may need an extra intermediate step to check the degrees of smoothness of coefficient functions. In practice it may not always be possible to correctly determine which function is the smoothest one. In such cases the the two-step method may lose its desired advantage over the conventional approach.

In this paper, we propose a new estimation method. We assign $h_{l}$ to estimate $g_{l}(\cdot)$, $\ell=1, \ldots, L$, so that we have $L$ bandwidths $\left(h_{1}, \ldots, h_{L}\right)$, one for each coefficient function. We minimize the following $\mathrm{CV}$ function by selecting $\left(h_{1}, \ldots, h_{L}\right)$ simultaneously

$$
C V\left(h_{1}, \ldots, h_{L}\right)=\frac{1}{n} \sum_{i=1}^{n}\left[Y_{i}-\sum_{\ell=1}^{L} \widehat{g}_{\ell, h_{\ell},-i}\left(Z_{i} ; 1\right) X_{i \ell}\right]^{2} .
$$


The difference between the our approach and the two-step procedure is that $h_{\ell}, \ell=$ $1, \ldots, L$, are selected simultaneously, we do not need to know which coefficient function is smoother than others. Also, unlike the conventional method that uses one smoothing parameter to estimate all coefficient functions (hence, some functions may be undersmoothed while others oversmoothed), we allow for different amount of smoothing for different coefficient functions.

\section{$3 \quad$ Numerical Examples}

In this section, we illustrate the finite sample performance of our proposed method in terms of estimation mean squared errors (MSE) defined by

$$
\frac{1}{n} \sum_{i=1}^{n}\left[\widehat{g}_{\ell}\left(Z_{i}\right)-g_{\ell}\left(Z_{i}\right)\right]^{2}, \ell=1, \ldots, L
$$

We consider the following functional coefficient model

$$
Y=g_{1}(Z) X_{1}+g_{2}(Z) X_{2}+U
$$

The covariates $\left(X_{1}, X_{2}\right)$ are generated from a standard bivariate normal distribution with correlation coefficient $2^{-1 / 2}, Z$ is uniformly distributed on $[0,1],\left(X_{1}, X_{2}\right)$ are independent of $Z, U$ follows normal distribution with mean zero and variance $\sigma^{2}$. Following Fan and Zhang (1999) we choose $\sigma^{2}=0.2 \cdot \operatorname{Var}\left(g_{1}(Z) X_{1}+g_{2}(Z) X_{2}\right)$ so that signal to noise ratio is about $5: 1$.

We first consider the following coefficient functions

$$
\text { Design } 1:\left\{\begin{array}{l}
g_{1}(z)=\sin (8 \pi(z-0.5)) ; \\
g_{2}(z)=3.5 \exp \left(-(4 z-1)^{2}\right)+3.5 \exp \left(-(4 z-3)^{2}\right)-1.5
\end{array}\right.
$$

This design is also used in Fan and Zhang (1999) and in Cai (2002, with logarithm as the link function), and satisfies the requirement that $g_{2}(\cdot)$ is smoother than $g_{1}(\cdot)$.

Second, the equally smoothed coefficient functions are used to illustrate the performance of our proposed estimator

$$
\text { Design } 2:\left\{\begin{array}{l}
g_{1}(z)=3.5 \exp \left(-(4 z-1)^{2}\right)+3.5 \exp \left(-(4 z-3)^{2}\right)-1.5 \\
g_{2}(z)=-3 \exp \left(-(4 z-.9)^{2}\right)-2.5 \exp \left(-(4 z-3)^{2}\right)+3.5
\end{array}\right.
$$

The coefficient functions $g_{1}(\cdot)$ and $g_{2}(\cdot)$ of Design 1 and Design 2 are displayed in Figure 1 .

For each of the above design, we conducted 2000 simulations with sample size $n=250$ and 500. For each simulation, the MSEs for $g_{1}(\cdot)$ and $g_{2}(\cdot)$ are computed. As argued in Fan and 
Zhang (1999), the two-step estimator is not very sensitive to the choice of initial bandwidth as long as it is small enough so that the bias in the first step smoothing is negligible. They suggest a simple automatic rule: use cross-validation or generalized cross-validation to select the bandwidth $h_{1}$ for the one-step fit. Then, use $h_{0}=0.5 h_{1}$ to undersmooth all, but the smoothest coefficient function at second stage. Cai (2002) suggests that one may use an even smaller $h_{0}$, say, $h_{0}=0.2 h_{1}$. To examine the sensitivity of different choice of $\alpha$ in $h_{0}=\alpha h_{1}$ for the two-step method, we report simulation results using both $\alpha=0.2$ and 0.5 . Also, in order to compare the same estimators for different methods of selecting bandwidths, we use local linear estimation method for all the simulations reported in this section.

The estimation results for Design 1 is reported in Table 1. From Table 1, we observe that the two-step method and our proposed method give similar MSE for $g_{1}(\cdot)$. This is understanable because the initial stage estimate of the two-step method give efficient estimation result for the less smoothed function $g_{1}(\cdot)$. For estimation of $g_{2}(\cdot)$, our proposed method has the smallest MSE, followed by the two-step method with $\alpha=0.5$. It seems that $\alpha=0.2$ undersmooth $g_{1}(\cdot)$ too much, its large estimated variance hurts the second stage $g_{2}(\cdot)$ estimate. When the sample size is doubled, all estimation MSEs reduce as expected.

Table 2 reports estimation results for design 2 where we observe that the results mirror that of Table 1 . While estimation results are similar for $g_{1}(\cdot)$, our proposed method give better estimation result for $g_{2}(\cdot)$, followed by the two-step method with $\alpha=0.5$.

\section{An Empirical Application}

In this section, we use the Canadian and U.S. exchange rate data to illustrate our estimation method. We use a similar data as that in Li et al. (2015) who investigate the purchasing power parity (PPP) hypothesis using Canadian and US price and exchange rate data. The PPP theory is typically tested under the following linear model setup.

$$
s_{t}=\alpha_{0}+\alpha_{1} p_{t}+\alpha_{2} p_{t}^{*}+u_{t},
$$

where $s_{t}, p_{t}$, and $p_{t}^{*}$ are the logarithm of the nominal exchange rate expressed as Canadian dollar per unit of US dollar, Canadian and the U.S. aggregate price levels, respectively. The PPP theory predicts that $\alpha_{0}=0, \alpha_{1}=1, \alpha_{2}=-1$ and $u_{t}$ is an $I(0)$ variable. However, most existing empirical studies reject the PPP theory based on linear model specifications. Li et al. (2015) suggest using a semiparametric varying model to investigate the PPP theory.

We have monthly data from January 1974 to December 2009. The aggregate price index is proxied by the producer price index (PPI) instead of the CPI. The PPI values are raw numbers with year 2000 as the base year. All the data was obtained from DataStream. Specif-

ically, we will use the yield spread differentials between the two countries as an additional 
covariate to help explain exchange rate movement. We downloaded monthly observations on 3 -month treasury bill rate $\left(T_{3, t}\right)$ and 10-year treasury bond rate $\left(T_{10, t}\right)$ data for both Canada and US from DataStream. Then, denote $z_{t}=\left(T_{10, t, C A}-T_{3, t, C A}\right)-\left(T_{10, t, U S}-T_{3, t, U S}\right)$ to be the yield spread differential between Canada and US. Allowing the coefficients in model (5) to vary with respect to the yield spread differentials, we fit the data by the following semiparametric varying coefficient model

$$
s_{t}=g_{0}\left(z_{t}\right)+g_{1}\left(z_{t}\right) p_{t}+g_{2}\left(z_{t}\right) p_{t}^{*}+u_{t} .
$$

Model (6) is motivated by the fact that monetary policy changes are made to respond to real economic activities and the yield spread between the 10-year treasury bond rate and 3-month treasury bill rate is considered to be a leading indicator of the real economic activity as explained in Estrella and Hardouvelis (1991).

Introducing yield spread differentials implies that the movements of exchange rates are affected by market's aggregate expectation of the two countries' relative pace of real economic developments. The market's expectation is adjusted quickly with respect to the release of relevant economic news (as reflected by the change of yield spread differential), and the bilateral exchange rate varies accordingly. Therefore, allowing the coefficients to change with the yield spread differentials can better explain the short-run movements of the bilateral exchange rates.

Figure 2 plots the nonparametric estimates of $g_{1}(\cdot)$ and $-g_{2}(\cdot)$ (negative of $g_{2}(\cdot)$ ) from our new method (CHL) and the existing two step method (FZ) respectively. We find that the estimated coefficient curves $\left(g_{1}(z)\right.$ and $\left.-g_{2}(z)\right)$ from two different methods exhibit similar shapes but our method offering smoother estimate for $g_{2}(\cdot)$ when the yield spread differentials are negative and large. The two estimation methods have similar computation cost. For this empirical data application, using a personal desktop with CORE 2 with $3.14 \mathrm{GHz}$ dual processors, the computation time for FZ method is 65.3 seconds while it is 67.81 seconds using our proposed method.

\section{Conclusion}

In this paper, we propose a new method of selecting smoothing parameters in estimating the coefficient functions in a functional-coefficient model. We assign a different bandwidth to different function and we select all the bandwidths simultaneously. The proposed method avoids the conventional approach which may undersmooth some functions while oversmooth other functions. It also avoids deciding which function is the smoothest one as is required in the two-step estimation method. Simulation results show that our proposed method performs well compared with existing methods. 


\section{References}

Cai, Zongwu, 2002, Two-step likelihood estimation procedure for varying-coefficient models, Journal of Multivariate Analysis 82(1), 189-209.

- 2007, Trending time-varying coefficient time series models with serially correlated errors, Journal of Econometrics 136(1), 163-188.

Cai, Zongwu, Jianqing Fan, and Runze Li, 2000, Efficient estimation and inferences for varying-coefficient models, Journal of the American Statistical Association 95(451), 888902.

Cai, Zongwu, Mitali Das, Huaiyu Xiong, and Xizhi Wu, 2006, Functional coefficient instrumental variables models, Journal of Econometrics 133(1), 207-241.

Card, David, 2001, Estimating the return to schooling: Progress on some persistent econometric problems, Econometrica 69(5), 1127-1160.

Cleveland, William S, Eric Grosse, and William M Shyu, 1992, Local regression models, Statistical models in S 2, 309-376.

Estrella, Arturo, and Gikas A Hardouvelis, 1991, The term structure as a predictor of real economic activity, The journal of Finance 46(2), 555-576.

Fan, Jianqing, and Wenyang Zhang, 1999, Statistical estimation in varying coefficient models, Ann. Statist. 27(5), 1491-1518.

Hastie, Trevor, and Robert Tibshirani, 1993, Varying-coefficient models, Journal of the Royal Statistical Society. Series B (Methodological) 757-796.

Juhl, Ted, 2005, Functional-coefficient models under unit root behaviour, The Econometrics Journal 8(2), 197-213.

Li, Hongjun, Zhongjian Lin, and Cheng Hsiao, 2015, Testing purchasing power parity hypothesis: a semiparametric varying coefficient approach, Empirical Economics 48(1), 427438.

Li, Qi, Cliff J Huang, Dong Li, and Tsu-Tan Fu, 2002, Semiparametric smooth coefficient models, Journal of Business \& Economic Statistics 20(3), 412-422.

Sun, Yiguo, and Qi Li, 2011, Data-driven bandwidth selection for nonstationary semiparametric models, Journal of Business \& Economic Statistics 29(4), 541-551. 
Xiao, Zhijie, 2009, Functional-coefficient cointegration models, Journal of Econometrics $152(2), 81-92$. 

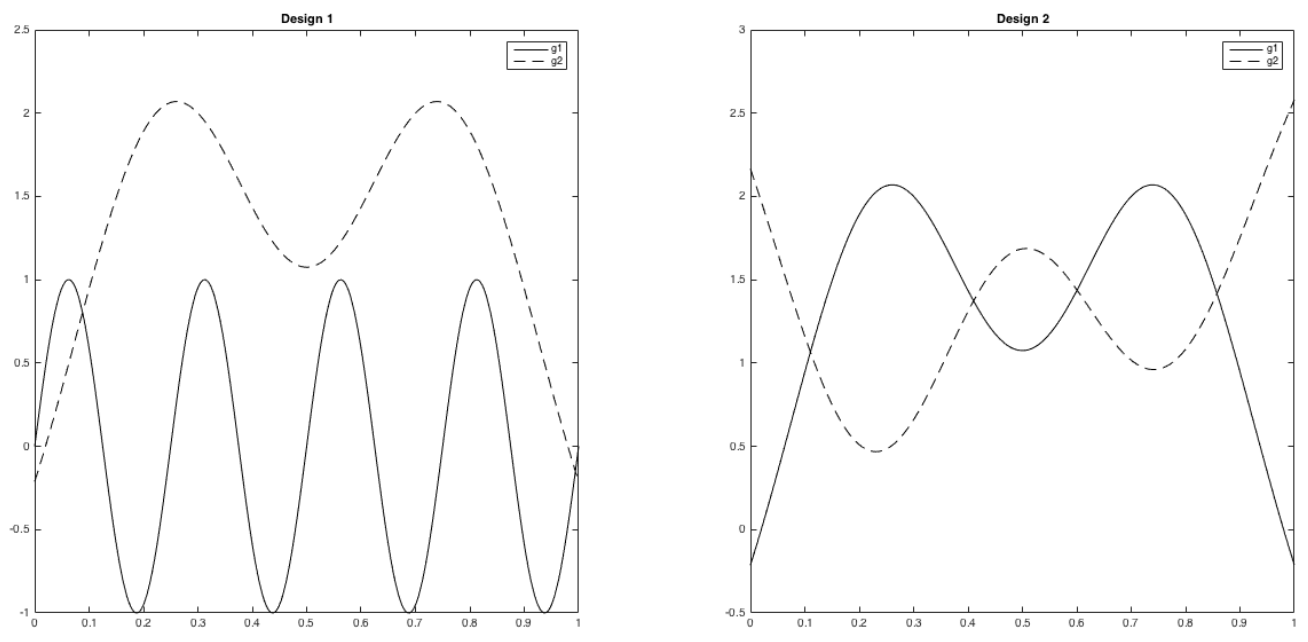

Figure 1: Coefficient Functions: The solid curve is $g_{1}(\cdot)$ and the dashed line is $g_{2}(\cdot)$.

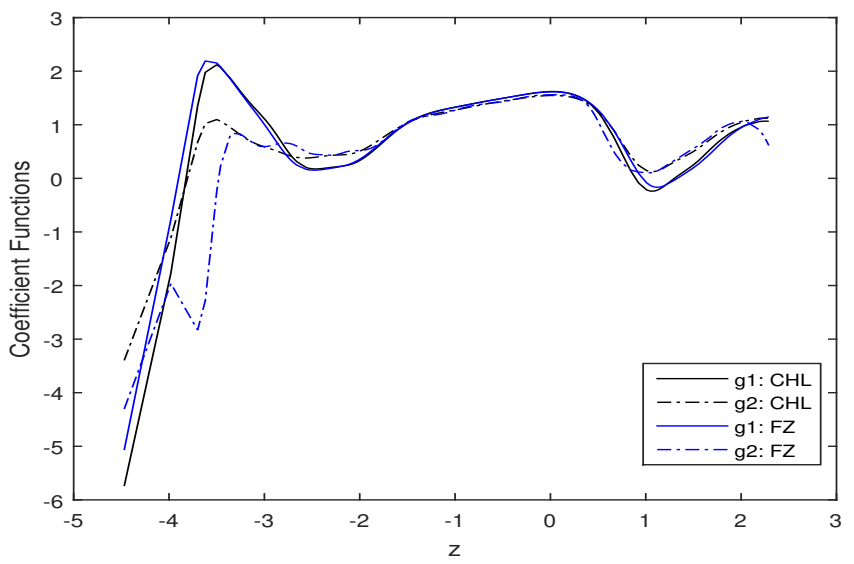

Figure 2: Coefficient Estimates for $g_{1}(\cdot)$ and $g_{2}(\cdot)$ 
Table 1: Simulation results for Design 1: Numbers in the row of $\tau$ represent the $\tau$ th percentile of the 2000 mean squared error values.

\begin{tabular}{cc|ccc|ccc}
\hline \multirow{2}{*}{$g_{\ell}(\cdot)$} & $\tau$ & \multicolumn{4}{|c|}{$n=250$} & \multicolumn{3}{c}{$n=500$} \\
& & $\begin{array}{c}\text { The proposed } \\
\text { method }\end{array}$ & $\begin{array}{c}\text { Two-step } \\
\alpha=0.2\end{array}$ & $\begin{array}{c}\text { Two-step } \\
\alpha=0.5\end{array}$ & $\begin{array}{c}\text { The proposed } \\
\text { method }\end{array}$ & $\begin{array}{c}\text { Two-step } \\
\alpha=0.2\end{array}$ & $\begin{array}{c}\text { Two-step } \\
\alpha=0.5\end{array}$ \\
\hline \multirow{3}{*}{$g_{1}(\cdot)$} & 25 & 0.0430 & \multicolumn{2}{c|}{0.0425} & 0.0228 & 0.0226 \\
& 50 & 0.0540 & \multicolumn{2}{c|}{0.0538} & 0.0282 & 0.0279 \\
& 75 & 0.0685 & \multicolumn{2}{c|}{0.0685} & 0.0343 & 0.0342 \\
\hline \multirow{3}{*}{$g_{2}(\cdot)$} & 25 & 0.0257 & 0.0401 & 0.0326 & 0.0131 & 0.0245 & 0.0172 \\
& 50 & 0.0356 & 0.0773 & 0.0485 & 0.0175 & 0.0387 & 0.0258 \\
& 75 & 0.0485 & 0.1257 & 0.0736 & 0.0241 & 0.0493 & 0.0375 \\
\hline
\end{tabular}

Table 2: Simulation results for Design 2: Numbers in the row of $\tau$ represent the $\tau$ th percentile of the 2000 mean squared error values.

\begin{tabular}{|c|c|c|c|c|c|c|c|}
\hline \multirow[b]{2}{*}{$g_{\ell}(\cdot)$} & \multirow[b]{2}{*}{$\tau$} & \multicolumn{3}{|c|}{$n=250$} & \multicolumn{3}{|c|}{$n=500$} \\
\hline & & $\begin{array}{c}\text { The proposed } \\
\text { method }\end{array}$ & $\begin{array}{c}\text { Two-step } \\
\alpha=0.2\end{array}$ & $\begin{array}{c}\text { Two-step } \\
\alpha=0.5\end{array}$ & $\begin{array}{c}\text { The proposed } \\
\text { method }\end{array}$ & $\begin{array}{c}\text { Two-step } \\
\alpha=0.2\end{array}$ & $\begin{array}{c}\text { Two-step } \\
\alpha=0.5\end{array}$ \\
\hline \multirow{3}{*}{$g_{1}(\cdot)$} & 25 & 0.0667 & \multicolumn{2}{|c|}{0.0662} & 0.0361 & \multicolumn{2}{|c|}{0.0367} \\
\hline & 50 & 0.1000 & \multicolumn{2}{|c|}{0.0968} & 0.0528 & \multicolumn{2}{|c|}{0.0526} \\
\hline & 75 & 0.1453 & \multicolumn{2}{|c|}{0.1393} & 0.0751 & \multicolumn{2}{|c|}{0.0754} \\
\hline \multirow{3}{*}{$g_{2}(\cdot)$} & 25 & 0.0623 & 0.0879 & 0.0643 & 0.0343 & 0.0513 & 0.0366 \\
\hline & 50 & 0.0939 & 0.1490 & 0.0971 & 0.0503 & 0.0838 & 0.0519 \\
\hline & 75 & 0.1465 & 0.2363 & 0.1467 & 0.0706 & 0.1314 & 0.0759 \\
\hline
\end{tabular}

